\title{
IMPUESTOS DIRECTOS E INDIRECTOS EN LA ARGENTINA: SU RELACIÓN CON EL DESEMPEÑO ECONÓMICO*
}

\author{
Cintia Martínez**
}

enviado: marzo 2016 - aceptado: junio 2016

\begin{abstract}
Resumen
El vínculo entre las instituciones políticas, las decisiones fiscales y los resultados económicos es materia de investigación desde hace unos años, especialmente a partir de los desarrollos teóricos relacionados, en primer término, con los modelos de crecimiento endógeno que dan espacio para las acciones de política económica y, posteriormente, con los aportes de la teoría de las decisiones públicas y del enfoque de la nueva economía institucional a las finanzas públicas. Las predicciones de la teoría son contundentes: las instituciones, ya sean políticas, económicas o fiscales, importan. Mediante el uso de modelos de vectores autorregresivos se explora la relación entre los impuestos directos e indirectos y el desempeño de la Argentina durante los últimos 80 años.
\end{abstract}

Código JEL: C5, E62.

Palabras clave: VAR, estructura impositiva, Argentina

\begin{abstract}
The connection between political institutions, fiscal decisions and economic results has been a subject of study during the last years, especially after the theoretical developments related first with endogenous growth models and, later, with the new paradigms of the Public Choice Theory and contributions of the New

* Este trabajo forma parte de la tesis doctoral de la autora, Instituciones Políticas, Decisiones Fiscales y Resultados Económicos: el Caso de Argentina.

** Departamento de Economía, Universidad Nacional del Sur, cmartinez@uns.edu.ar
\end{abstract}


Institutional Economy to public finance. The theoretical predictions are sound: political, economic and fiscal institutions matter. We explore, using vector autoregressive models, the relation between direct and indirect taxes and the economic performance of Argentina in the last 80 years.

JEL Code: C5, E62.

Keywords: VAR, tax structure, Argentina.

\section{INTRODUCCIÓN}

Los tributos constituyen un tema de preocupación de todos los tiempos, a tal punto que incluso los primeros estudiosos en finanzas públicas se preocupan mucho por las consecuencias que la fiscalidad podría tener sobre la prosperidad de las naciones.

A partir del desarrollo de los modelos de crecimiento endógeno que incorporan en su planteo aspectos relacionados con el accionar del sector público e instituciones fiscales, se empieza a clarificar que las acciones estatales pueden alterar las decisiones de los agentes económicos y, por esa vía, afectar las fuentes de crecimiento. Consecuentemente, el estudio de las diferentes instituciones fiscales y su influencia sobre el desempeño económico cobra un gran impulso.

Numerosos desarrollos teóricos y abundantes trabajos empíricos, realizados en su mayoría para grupos de países desarrollados, ponen de manifiesto cierta discordancia entre las predicciones de la teoría económica y los hallazgos de los trabajos aplicados. La evidencia empírica existente hasta el momento no alcanza a confirmar la teoría, sus resultados no son concluyentes. Estos hechos son la fuente de inspiración de esta investigación que intenta contribuir a la discusión mediante un análisis aplicado a la economía argentina.

El objetivo del presente trabajo es estudiar la relación entre la imposición directa e indirecta y los resultados económicos para el caso argentino. Los impuestos pueden ser considerados como instituciones. Son normas que establecen "beneficios" o "costos" para determinados comportamientos de la gente. Por lo tanto, forman parte del sistema de incentivos e influyen sobre la conducta de las personas que toman decisiones económicas. Es a través de esta vía que el diseño del sistema impositivo puede afectar el desempeño económico de una nación. 
El trabajo se organiza de la siguiente manera: en primer lugar, se describe el marco teórico y la metodología empleada, luego se especifica empíricamente el modelo planteado y se describe el diagnóstico econométrico realizado para garantizar la validez de los resultados. Finalmente, se expone el modelo y sus resultados.

\section{MARCO TEÓRICO Y METODOLOGÍA}

Una de las herramientas, entre tantas disponibles, que puede ser de utilidad para analizar las relaciones entre las instituciones fiscales argentinas y el desempeño económico del país es la técnica de modelos de vectores autorregresivos, en adelante modelos VAR (Sims, 1980) ${ }^{1}$. Se utiliza este tipo de instrumento cuando se pretende caracterizar y comprender las interacciones simultáneas que pueden existir en un conjunto de variables, en este caso, variables económicas. La técnica permite estimar el efecto que un cambio o shock en una de las variables tiene sobre las demás sin perder de vista la retroalimentación que existe entre las mismas. Son más sencillos de estimar y permiten la predicción a través del análisis de las funciones impulso respuesta (FIR).

Las propiedades descriptas hacen que este enfoque resulte adecuado para el estudio de las instituciones fiscales y el desempeño económico toda vez que, frente a cambios fiscales, transcurre un lapso de tiempo hasta que se visualizan sus efectos sobre el producto. Se considera que esta técnica es superadora de las estrategias de análisis econométrico más tradicional, aun cuando persisten en ella algunas falencias al momento de explicar las relaciones subyacentes en el vínculo entre la fiscalidad y los sistemas económicos.

La mayoría de las aplicaciones del modelo VAR no se relaciona con el campo de las finanzas públicas y cuando sí lo hacen estudian los fenómenos fiscales de economías como la de Estados Unidos o de países europeos, sin focalizarse en economías emergentes o en desarrollo. Peor aún, los estudios existentes no se concentran en economías como la argentina, con niveles históricos de déficit fiscal altos y casi permanentes, además de ser corriente el uso excesivo tanto del crédito público como de la emisión monetaria como forma de financiación de los mismos.

1 El modelo VAR se aplicó al estudio de la macroeconomía originalmente por Christopher Sims (1980), quien en 2011 fue reconocido con el premio Nobel por sus aportes en este campo. En el área de las finanzas públicas se aplica desde hace unos años investigando los efectos de la política fiscal sobre el producto. 
Por otra parte, en general, la evidencia empírica disponible hasta el momento basada en la metodología VAR evalúa, en conjunto, los gastos públicos y la recaudación tributaria, analizando los efectos globales de la acción estatal sobre la economía sin discriminar mayormente entre diferentes clases de tributos o de gastos públicos. Incluso, en el campo de las finanzas públicas, se encuentra mayor cantidad de trabajos donde se profundiza el análisis de los diferentes componentes del gasto público y su impacto sobre el crecimiento, en comparación con la bibliografía empírica relacionada con aspectos de la tributación.

Entre los antecedentes principales en la materia no puede dejar de mencionarse el trabajo de Blanchard y Perotti (2002) y el posterior aporte de Perotti (2004). Como ya se ha señalado, los resultados no son concluyentes.

En la Argentina las aplicaciones de la metodología VAR no abundan y, con frecuencia, se ocupan de cuestiones ajenas a la economía pública. En tal sentido, el trabajo de Rezk, Avramovich, y Basso (2006) constituye un gran aporte al campo de las finanzas públicas al estudiar el vínculo entre las instituciones fiscales y el producto interno bruto, la tasa de inflación y el desempleo en Argentina. Las variables fiscales que toman los autores son el gasto público corriente y los recursos tributarios, ambos incluyen el nivel nacional y provincial. También, consideran el producto interno bruto, el desempleo y la tasa de inflación. Encuentran que al incrementarse los recursos tributarios el producto interno bruto reacciona negativamente, mientras que ante un shock en el gasto público el producto se incrementa inicialmente y luego aparecería un indicio de efecto crowding out. En líneas generales, los resultados son sorprendentemente mínimos y, en muchos casos, no resultan significativos.

\section{ESPECIFICACIÓN EMPÍRICA, TRATAMIENTO DE LAS VARIABLES Y DIAGNÓSTICO ECONOMÉTRICO DE LOS MODELOS VAR}

En función del objetivo del trabajo, que consiste en evaluar el efecto que tienen sobre el producto interno bruto las variaciones en el indicador tributario que relaciona los impuestos directos y los indirectos, se plantea un modelo VAR intentando conseguir la configuración más parsimoniosa, pero al mismo tiempo tratando de evitar el sesgo de variables omitidas. Debe aclararse que aquí se pone especial atención sobre aspectos de eficiencia, y no así de equidad, dado que no se estudia el impacto de las modificaciones impositivas sobre cuestiones distributivas.

Dada la enorme cantidad de información disponible referida a las instituciones fiscales argentinas, en la práctica es necesario construir nuevas variables 
que las agrupen alternativamente en función del objetivo de análisis, en este caso la relación entre impuestos directos e indirectos y, a partir de allí, hacer posible el planteo de un modelo.

Para la especificación del modelo planteado en el presente trabajo es indispensable cumplir con las siguientes etapas ${ }^{2}$, cuyos resultados se encuentran disponibles en el anexo A:

- Análisis de la estacionariedad de las series y determinación del orden de integración: se utilizan los test de Dickey-Fuller Aumentado y Phillips-Perron para las variables en nivel y en primeras diferencias.

- Análisis de quiebres estructurales: se aplica el test de Zivot-Andrews.

- Planteo del modelo VAR: organización de las variables y estimación del VAR, incluyendo componentes determinísticos o no en virtud de su significatividad.

- Contrastes de especificación: establecimiento del número de retardos más aconsejable de modo de garantizar la eliminación de la autocorrelación serial de los residuos siguiendo los criterios de información habituales contenidos en los paquetes econométricos: Schwarz, Akaike y Hannan-Quinn, principalmente.

- Análisis de los residuos y de la estabilidad del VAR: es necesario confirmar que los residuos del modelo estimado son de tipo "ruido blanco". Adicionalmente, se debe comprobar la estabilidad del modelo VAR para garantizar que no existe un comportamiento explosivo descartando así la presencia de raíces unitarias en su representación de media móvil. En caso de obtener un VAR bien comportado se decide plantearlo con las variables en niveles y no en diferencias para no perder información de largo plazo. Posteriormente, se estudia la hipótesis de cointegración en cuyo caso se especifica un modelo de corrección de error.

- Estimación de la FIR: estas funciones recogen los impactos producidos por los shocks sorpresivos, de un desvío estándar, en las variables del sistema. Se sigue el procedimiento habitual de la literatura y se escoge la descomposición de Cholesky de la matriz de varianzas y covarianzas de los residuos estimados, que solo requiere imponer algunas restricciones sobre las relaciones contemporáneas de las variables. Se elige esta descomposición debido a que el interés de la investigación es describir la dinámica conjunta de las variables y no hacer pronósticos.

2 El análisis econométrico se efectúa totalmente con el EViews 8. Solo en el caso del test de raíz unitaria en presencia de quiebre estructural, Zivot- Andrews, se utiliza el paquete Stata 12.0. 
- Análisis de descomposición de varianza: calcula la contribución de cada shock estructural al error de predicción de cada variable por una cierta cantidad de períodos hacia delante. Tal como se comenta anteriormente se trata de un análisis complementario de la FIR que contribuye además a dar respaldo al orden impuesto a las variables del VAR.

\section{ESPECIFICACIÓN DEL MODELO Y PRESENTACIÓN DE LOS RESULTADOS}

La frecuencia de los datos es anual, abarcando el período 1932 a 2013. La elección del período se justifica porque, hasta los años treinta, Argentina tiene un sistema impositivo estructuralmente muy diferente al que comienza a gestarse a partir de 1932 y que, en rasgos generales, perdura hasta nuestros días. Hasta ese momento existe un predominio de impuestos indirectos, especialmente recursos provenientes del comercio internacional e impuestos sobre consumos específicos. Luego, desde 1932 en adelante, el sistema impositivo se estructura sobre un impuesto a los ingresos y un gravamen al consumo, a los que se agregan algunos impuestos al comercio exterior, otros sobre bases patrimoniales y también sobre cierto tipo de transacciones. Tanto en el caso del gasto público como en el de los recursos tributarios los datos corresponden al nivel nacional.

La serie de producto interno bruto, PIB, se encuentra expresada en valores constantes de 1993. Para el planteo del modelo se construyen variables fiscales ad hoc de acuerdo con el objetivo puntual de análisis.

Se parte de un modelo VAR planteado en niveles, aun habiendo encontrado que las series son no estacionarias, siempre que los mismos cumplan con las condiciones de estabilidad y que los residuos sean de tipo "ruido blanco". Si bien hay discusión al respecto en la literatura (Enders, 1995), el principal argumento a favor de la construcción del VAR en niveles se refiere a que al diferenciar las series se pierden los comovimientos en los datos temporales y las posibles relaciones de cointegración entre ellos. Incluso, el mismo Enders cita que Sims (1980) se opone a la diferenciación aun en presencia de raíz unitaria argumentando que la finalidad de los modelos VAR es la determinación de interrelaciones entre las variables y no así la estimación o el análisis de sus parámetros.

Un VAR es un sistema de dos o más series de tiempo que se modela considerando rezagos de las variables y la interacción dinámica que pudiera existir entre ellas. En general, un VAR de $k$ variables $y p$ rezagos se define de la siguiente manera: 


$$
y_{t}=\emptyset+\Pi_{1} y_{t-1}+\Pi_{2} y_{t-2}+\cdots+\Pi_{p} y_{t-p}+\varepsilon_{t},
$$

Donde

$\phi^{\prime}=\left(\phi_{1}, \phi_{2}, \ldots \phi_{k}\right)$ es un vector de $\mathrm{k}$ constantes,

$y_{t}^{\prime}=\left(y_{1 t}, y_{2 t}, \ldots y_{k t}\right)$ es un vector de k variables endógenas,

$\varepsilon_{t}^{\prime}=\left(\varepsilon_{1 t}, \varepsilon_{2 t}, \ldots \varepsilon_{k t},\right)$ es el término de perturbaciones aleatorias,

$\Pi_{p}=\left[\begin{array}{ccc}\Pi_{11 . p} & \cdots & \Pi_{1 k . p} \\ \vdots & \ddots & \vdots \\ \Pi_{k 1 . p} & \cdots & \Pi_{k k . p}\end{array}\right]$ es la matriz de coeficientes asociada al vector $\mathrm{y}$.

Cuando se elige trabajar con modelos de vectores autorregresivos expresados en niveles se puede estudiar posteriormente la posibilidad de cointegración entre las variables que lo componen y, en caso de encontrar resultados positivos, es posible estimar un modelo de vectores autorregresivos con corrección de errores, VECM.

Engle y Granger (1987) plantean el argumento que se conoce como teorema de representación de Granger. Muestran que cuando una combinación lineal de variables que son integradas de orden 1, I(1), puede representarse como una combinación lineal que es $\mathrm{I}(0)$, entonces ese conjunto de variables puede representarse como un modelo de corrección de error. Formalmente, puede escribirse del siguiente modo:

$$
\Delta y_{t}=\alpha z_{t-1}+\Gamma_{1} \Delta \mathrm{y}_{\mathrm{t}-1}+\cdots+\Gamma_{1} \Delta \mathrm{y}_{\mathrm{t}-(\mathrm{p}-1)}+\Phi(\mathrm{L}) \varepsilon_{\mathrm{t}}
$$

Donde representa los $\mathrm{r}$ vectores de cointegración. El número de rezagos del VAR restringido representado como VEC debe ser p-1, es decir un rezago menos que el óptimo seleccionado para el VAR, de modo de garantizar resultados confiables en su aplicación práctica.

Dos o más series cointegran si comparten una tendencia común, aun en aquellos casos donde las series sean no estacionarias, es decir, incluso cuando tengan tendencia estocástica. Cuando hay cointegración existe una combinación lineal estacionaria entre las variables, un equilibrio de largo plazo hacia el cual converge el sistema. Los coeficientes de integración representan los ponderadores que hacen que la combinación lineal entre las variables que cointegran sea estacionaria. El mecanismo de corrección de error es aquel que compatibiliza los comportamientos de corto plazo y de largo plazo de las variables y se interpreta como el ajuste hacia el equilibrio. 
III.1. Relación entre impuestos directos e indirectos

La intención es explorar el efecto que tiene sobre el producto una modificación en la composición entre tributos directos e indirectos en la Argentina. Con esa finalidad se construyen la variable TDIR — que corresponde a la suma de los impuestos sobre los ingresos de las personas, utilidades, ganancias de capital, sobre la propiedad y las contribuciones de la seguridad social — y la variable TINDIR que resulta de la suma de los impuestos sobre el gasto y al comercio internacionalLuego, a partir de estas dos nuevas variables, se construye la serie TAXMIX como el cociente entre los impuestos directos y los indirectos (TDIR/TINDIR).

EL VAR, en consecuencia, quedaría integrado por el gasto público (GP), el cociente entre impuestos directos e indirectos (TAXMIX), el producto interno bruto (PIB) y la recaudación (RT), en ese orden y con las series transformadas en logaritmo natural.

Por ser la variable más exógena se ubica en primer término a GP seguida de TAXMIX. Las decisiones legislativas, e incluso administrativas, relacionadas con las instituciones fiscales tales como la introducción o remoción de nuevos tributos, modificaciones en los elementos de los impuestos o variaciones en el método de liquidación de las obligaciones tributarias, modifican el marco institucional en el que se desempeñan los agentes económicos.

Según el enfoque teórico adoptado por esta investigación el marco institucional vigente determina el comportamiento de los individuos que son, en última instancia, quienes toman las decisiones privadas que terminan definiendo los resultados económicos. Como consecuencia, toda modificación en la estructura tributaria - como en el caso de este modelo la combinación entre impuestos directos e indirectos- puede impactar contemporáneamente sobre el nivel de producción.

Luego, corresponde incluir a PIB y por último a RT. Se estima un VAR con un solo rezago que, además de poseer residuos de tipo ruido blanco, satisface las condiciones de estabilidad requeridas.

En la figura 1 puede apreciarse que frente a un shock positivo en TAXMIX, es decir un incremento en la imposición directa en relación con la imposición indirecta, el PIB tiene una reacción negativa, aunque si se toma el intervalo de confianza del $95 \%$ resulta significativa solamente por un período. Si se tomara un intervalo de confianza menos exigente el efecto podría extenderse por un período de tiempo mayor. Para una correcta interpretación de los resultados debe recor- 
darse que un incremento en TAXMIX de ninguna manera implica un aumento en la carga de la imposición (ese efecto estaría captado por la variable RT), sino que se trata de un cambio en la composición relativa de la estructura tributaria.

El resultado encontrado está en línea con lo esperado dado que la teoría fiscal predice que los impuestos directos afectan relativamente más a los incentivos que la imposición indirecta $\mathrm{y}$, por consiguiente, desestimulan más la actividad económica.

Figura 1. Respuesta de PIB a TAXMIX

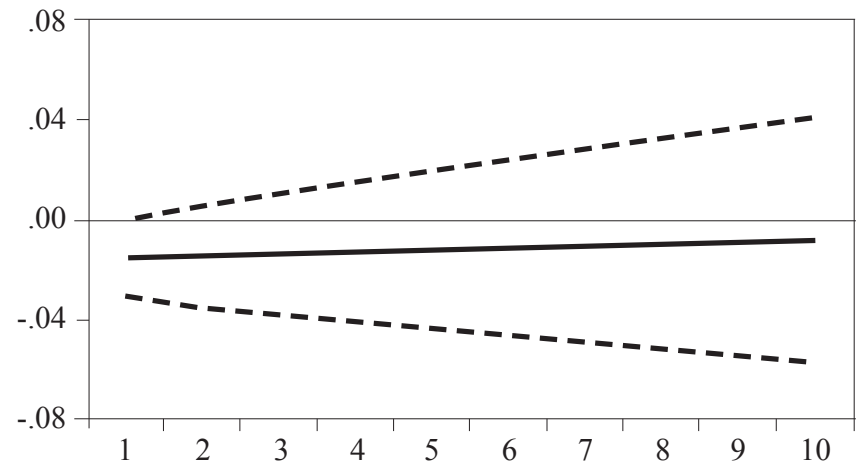

Fuente: elaboración propia.

Si se analiza el efecto que genera un incremento en el producto sobre las otras variables del modelo se verifica que ante un impulso positivo en el PIB el gasto público aumenta y la recaudación también lo hace, mientras que la relación entre impuestos directos e indirectos no responde a una innovación en el PIB bajo ningún intervalo de confianza (ver figura 2).

El análisis de descomposición de varianza, complementario al estudio de la FIR, está en línea con la presunción realizada acerca del orden recomendable para las variables.

El gasto público se presenta bastante exógeno al tener su varianza explicada básicamente por innovaciones propias y en muy menor medida por shocks en el PIB, mientras que ni los shocks en la recaudación ni en la composición tributaria entre impuestos directos e indirectos tienen influencia. 
Figura 2. Respuesta de TAXMIX a PIB

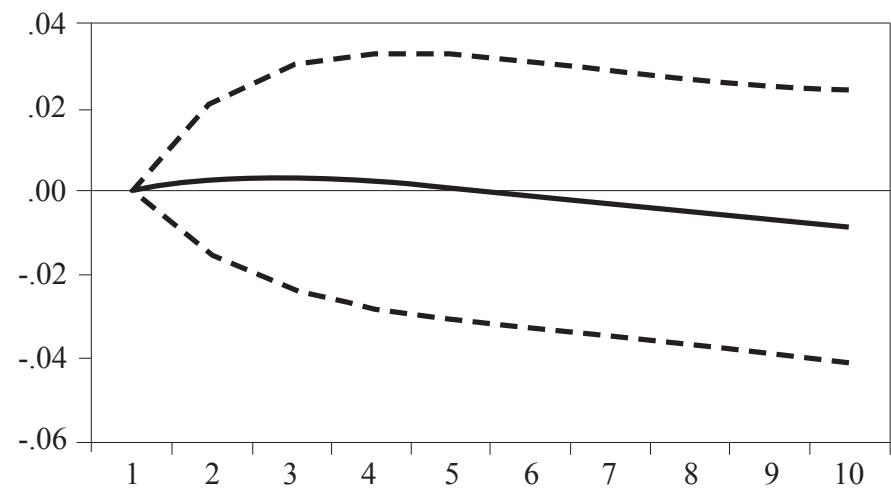

Fuente: elaboración propia.

En el caso del PIB la mayoría de su varianza se relaciona con su propio shock al mismo tiempo que una innovación en el gasto público también tiene una participación muy relevante en el primer momento, aunque rápidamente pierde peso con el transcurrir del tiempo. Un choque en la variable TAXMIX explica en el primer período algo más de un 3,5\% de la varianza del producto interno bruto.

Es interesante observar que el $90 \%$ de la varianza de TAXMIX se explica por shocks propios en el primer período, mientras que el resto es totalmente justificado por innovaciones en el gasto público, de modo tal que ni la recaudación ni el producto tienen participación. Esto podría indicar que es la decisión de gasto la que precede las modificaciones en la estructura tributaria.

III.2. Verificación de la hipótesis de cointegración

Al verificar la hipótesis de cointegración, el test de Johansen acepta la existencia de un vector de cointegración, por lo que se estima un modelo de corrección de error sin ningún rezago.

La ecuación de cointegración encontrada presenta coeficientes significativos para todas las variables y los signos son los esperados. La variable identificada con el número 1 es el gasto público de modo tal que está expresada como 
si fuera la variable dependiente. La relación entre el gasto público y el producto bruto es positiva así como también con la recaudación, mientras que la relación entre la variable que representa la estructura tributaria también exhibe una relación positiva con el gasto (ver tabla 1).

Todos los términos de corrección de error de la ecuación de cointegración son negativos, estadísticamente significativos y con valor absoluto menores que la unidad. Por ello, existe convergencia en el largo plazo. La mayor parte de la corrección hacia el equilibrio recae sobre $\mathrm{D}(\mathrm{GP})$, mientras que $\mathrm{D}(\mathrm{PIB})$ y $\mathrm{D}(\mathrm{RT})$ tienen coeficientes más pequeños. El coeficiente asociado a $\mathrm{D}(\mathrm{TAXMIX)}$ es el menor, por lo que puede concluirse que frente a un shock que saque al sistema de su equilibrio, el retorno al mismo no estaría fuertemente dominado por la variable asociada a la estructura tributaria. La corrección de este término existe, pero es lenta.

Tabla 1. Estimación del vector de corrección de error

\begin{tabular}{|c|c|c|c|c|}
\hline & CointEq1 & & & \\
\hline LNGP(-1) & 1.000000 & & & \\
\hline \multirow[t]{3}{*}{ LNTAXMIX(-1) } & -0.108990 & & & \\
\hline & $(0.04496)$ & & & \\
\hline & {$[-2.42396]$} & & & \\
\hline \multirow[t]{3}{*}{ LNPIB(-1) } & -0.925683 & & & \\
\hline & $(0.12758)$ & & & \\
\hline & {$[-7.25565]$} & & & \\
\hline \multirow[t]{3}{*}{ LNRT(-1) } & -0.123248 & & & \\
\hline & $(0.10365)$ & & & \\
\hline & [-1.98903] & & & \\
\hline \multirow[t]{3}{*}{$\mathrm{C}$} & 2.382689 & & & \\
\hline & $(0.60129)$ & & & \\
\hline & [3.96262] & & & \\
\hline Error Correction: & D(LNGP) & D(LNTAXMIX) & D(LNPIB) & $\mathrm{D}$ (LNRT) \\
\hline \multirow[t]{3}{*}{ CointEq1 } & -0.693423 & -0.096946 & -0.368160 & -0.470819 \\
\hline & $(0.09394)$ & $(0.10471)$ & $(0.06563)$ & $(0.12798)$ \\
\hline & [-7.38148] & [-1.98583] & {$[-5.60999]$} & {$[-3.67877]$} \\
\hline
\end{tabular}

Nota: Standard errors in ( ) \& t-statistics in [ ].

Fuente: elaboración propia. 
A partir de la ecuación de integración es posible despejar los coeficientes en función de variables de interés. En la tabla 2 se exhiben los resultados de la normalización. De allí surge que un incremento en el gasto público tiene una relación positiva con el PIB, mientras que un aumento en la recaudación genera una respuesta negativa en el producto. Es interesante destacar que un aumento en la variable TAXMIX, que indica una mayor proporción de impuestos directos en relación con impuestos indirectos, repercute negativamente en el PIB aunque el efecto no es demasiado importante con un coeficiente relativamente pequeño.

Con respecto a lo que ocurre con la variable TAXMIX, un incremento en el producto interno bruto generaría una caída en la proporción de impuestos directos en relación con los indirectos. Esto podría explicarse por la diferencia de elasticidad de los impuestos directos e indirectos con respecto a la base imponible y también con la eficacia recaudatoria relativa entre estas dos clases de impuestos ${ }^{3}$. Asociado con esto, un incremento en la recaudación conlleva una caída en la proporción de impuestos directos con relación a los indirectos.

Tabla 2. Normalización de los coeficientes de integración

\begin{tabular}{cccc}
\hline LNGP & LNTAXMIX & LNPIB & LNRT \\
\hline-1.08 & 0.12 & 1 & 0.13 \\
-9.18 & 1 & 8.49 & 1.13 \\
\hline
\end{tabular}

Fuente: elaboración propia

\section{CONCLUSIONES}

Aún con sus grandes limitaciones, el método VAR y el análisis de cointegración aparecen como la estrategia más adecuada para el objetivo de este trabajo. Dadas las peculiaridades de la economía argentina, reconocidas ampliamente por investigadores de nuestro país y del mundo, efectuar comparaciones con otros países resulta complicado.

Adicionalmente, no existe disponibilidad de información tributaria de otros países semejantes a la Argentina con el suficiente grado de desagregación que se necesitaría para la construcción del indicador de estructura tributaria. De allí que

3 Rezk et al. (2006) destacan la baja elasticidad ingreso de la recaudación impositiva en la Argentina. 
el uso de las series temporales correspondientes únicamente a la Argentina aparece como la elección más plausible.

El aporte del análisis de cointegración resulta sumamente enriquecedor porque permite diferenciar los efectos de corto plazo de los de largo plazo. Ratifica las conclusiones a las que se arriba luego de estimar el VAR.

Sintéticamente, las principales conclusiones que surgen del estudio realizado indican que un incremento en el gasto público total genera una reacción positiva en el PIB que es de carácter limitado y transitorio, ya que enseguida se produce un efecto negativo sobre el mismo, lo que puede tomarse como un indicio de un efecto desplazamiento en la actividad privada. Este resultado se obtiene en el VAR y es ratificado por el análisis de descomposición de la varianza del PIB, donde se ve que el efecto sobre el nivel de actividad es limitado, y también por el análisis de cointegración.

Por otra parte, aumentos en la recaudación tributaria total provocan una caída en el producto bruto y, si bien este efecto no resulta significativo, es ratificado por el signo de los ponderadores de largo plazo que surgen en el análisis de cointegración. Esto podría corresponderse con un modelo de economía de la oferta donde un aumento en la presión tributaria generaría una caída en la producción.

La reacción del gasto público frente a un aumento en el producto interno bruto es positiva según lo encontrado por el método VAR. Debe destacarse que no es objeto de estudio el gasto público en sí mismo ni su composición, simplemente se informan los resultados generales que no permiten, sin efectuar análisis adicionales, ser conclusivos al respecto. Adicionalmente, la representación de la ecuación de cointegración muestra que el gasto público tiene una relación positiva con el producto y con la recaudación, aunque en este último caso el coeficiente asociado es pequeño y de baja significatividad estadística.

Los resultados hallados podrían interpretarse como evidencia del comportamiento procíclico que históricamente ha demostrado el gasto público en la Argentina, revelando también la existencia de elementos compatibles con las predicciones de la teoría de las decisiones públicas. Contrariamente, el gasto público casi no reacciona frente a un aumento en la recaudación, respaldando la idea de que es el gasto la variable que impulsa un mayor esfuerzo para la obtención de ingresos tributarios y no al revés. Se verifica la mayor exogeneidad de la variable GP, es decir que no sería el gasto el que se adecúa a los recursos disponibles. Esto podría explicarse por el recurrente uso del déficit fiscal financiado 
con emisión monetaria y deuda pública, instrumentos que alternativamente han agotado los diferentes gobiernos.

Con respecto a la influencia de la relación entre impuestos directos e indirectos sobre el desempeño del producto puede afirmarse que un aumento en la imposición directa en relación con los tributos indirectos desestimula el nivel de actividad, al menos por un período (un año). Estas conclusiones del análisis del VAR son ratificadas por la ecuación de cointegración que muestra una relación inversa entre el PIB y el cociente entre impuestos directos e indirectos.

Al analizar el mecanismo de corrección de error, los resultados indican que frente a un shock que saque al sistema del equilibrio, la variable fiscal tiene una influencia muy pequeña al momento de recobrarlo. La velocidad de ajuste es lenta. También surge del análisis que un incremento en el producto tiene un efecto negativo sobre la variable TAXMIX, es decir que reaccionarían más los impuestos indirectos que los directos, lo que podría presumirse consecuencia de las diferentes elasticidades ingreso en las dos clasificaciones de impuestos.

\section{ANEXO A}

\section{A.1. Series utilizadas y fuentes de información}

- LNGP: gasto público en valores constantes de 1993, transformada a logaritmo natural. Fuente: Orlando Ferreres.

- LNPIB: producto interno bruto expresado en valores constantes de 1993, transformada a logaritmo natural. Fuente: Ministerio de Economía de la Nación y Ariel Coremberg.

- LNRT: recaudación total en valores constantes de 1993, transformada a logaritmo natural. Fuente: Estadísticas Tributarias de AFIP.

- LNTAXMIX: relación entre impuestos directos, TDIR, e indirectos, TINDIR. Transformada a logaritmo natural. La variable TDIR agrupa los impuestos sobre la renta, utilidades, ganancias de capital, propiedad y contribuciones al sistema de seguridad social. La variable TINDIR es la suma de los impuestos sobre el gasto y el comercio internacional. Las series originales de recaudación se encuentran expresadas en valores históricos y por tratarse de cocientes no es necesaria su conversión a pesos corrientes ni constantes. Fuente: Estadísticas Tributarias de AFIP. 
A.2. Tests de raíz unitaria y cambio estructural

ADF

\begin{tabular}{lcccc}
\hline & \multicolumn{2}{c}{ Nivel } & \multicolumn{2}{c}{ Primera diferencia } \\
\hline Variable & intercepto & $\begin{array}{c}\text { intercepto y } \\
\text { tendencia }\end{array}$ & intercepto & $\begin{array}{c}\text { intercepto y } \\
\text { tendencia }\end{array}$ \\
\hline LNGP & $-0,32$ & $-4,1$ & $-7,53$ & $-7,51$ \\
LNPIB & $-1,29$ & $-2,28$ & $-8,45$ & $-8,47$ \\
LNRT & $-0,81$ & $-2,33$ & $-9,2$ & $-9,14$ \\
LNTAXMIX & $-1,98$ & -2 & $-8,18$ & $-8,19$ \\
\hline
\end{tabular}

Critero Schwarz

\section{PHILLIPS PERRON}

\section{Nivel}

\begin{tabular}{lcccc} 
Variable & intercepto & $\begin{array}{c}\text { intercepto y } \\
\text { tendencia }\end{array}$ & intercepto & $\begin{array}{c}\text { intercepto y } \\
\text { tendencia }\end{array}$ \\
\hline GP & $-0,41$ & $-3,51$ & $-7,50$ & $-7,47$ \\
PBI & $-1,34$ & $-2,28$ & $-8,44$ & $-8,47$ \\
REC & $-0,79$ & $-2,43$ & $-9,20$ & $-9,14$ \\
TAXMIX & $-2,10$ & $-2,09$ & $-8,26$ & $-8,26$ \\
\hline
\end{tabular}

ZIVOT ANDREWS

\begin{tabular}{lccc}
\hline Variable & Retardos & Intercepto & Año \\
LNGP & 1 & $-4,37$ & 1984 \\
LNPIB & 0 & $-4,12$ & 1981 \\
LNRT & 1 & $-4,24$ & 1991 \\
LNTAXMIX & 0 & $-2,63$ & 1974 \\
\hline
\end{tabular}

Criterio de selección de retardos BIC

\begin{tabular}{lccc}
\hline Variable & Retardos & Tendencia & Año \\
\hline LNGP & 1 & $-4,16$ & 1975 \\
LNPIB & 0 & $-3,27$ & 1970 \\
LNRT & 1 & $-4,00$ & 1989 \\
LNTAXMIX & 0 & $-2,94$ & 1994 \\
\hline
\end{tabular}




\begin{tabular}{lccc}
\hline Variable & Retardos & Ambos & Año \\
\hline LNGP & 1 & $-4,26$ & 1947 \\
LNPIB & 0 & $-4,34$ & 1981 \\
LNRT & 1 & $-4,25$ & 1981 \\
LNTAXMIX & 0 & $-2,16$ & 1944 \\
\hline
\end{tabular}

\section{A.3 Criterios de longitud de rezagos}

VAR Lag Order Selection Criteria

Endogenous variables: LNGP LNTAXMIX LNPIB LNRT

Exogenous variables: C DUM2002 DUM1989

Date: 03/09/15 Time: 15:36

Sample: 19332013

Included observations: 81

\begin{tabular}{ccccccc}
\hline Lag & LogL & LR & FPE & AIC & SC & HQ \\
\hline 0 & $-29,42783$ & NA & 0,0000423 & 1,279657 & 1,789557 & 1,482428 \\
& & & $1.62 \mathrm{e}-$ \\
1 & 265,9471 & 524,1866 & $08 *$ & $-6,59006$ & & $-6.18451^{*}$ \\
2 & 279,3431 & 22,2637 & $1,76 \mathrm{E}-08$ & $-6,516707$ & $-4,987008$ & $-5,908394$ \\
& & & & & & \\
3 & 298,2983 & $29.36724 *$ & $1,65 \mathrm{E}-08$ & $-6.599952^{*}$ & $-4,560353$ & $-5,788868$ \\
4 & 309,2871 & 15,7867 & $1,96 \mathrm{E}-08$ & $-6,458791$ & $-3,909293$ & $-5,444936$ \\
5 & 315,4605 & 8,173278 & $2,71 \mathrm{E}-08$ & $-6,181986$ & $-3,122588$ & $-4,965361$ \\
6 & 323,4618 & 9,691639 & $3,64 \mathrm{E}-08$ & $-5,956669$ & $-2,387371$ & $-4,537273$ \\
7 & 332,0624 & 9,448595 & $4,95 \mathrm{E}-08$ & $-5,748237$ & $-1,669039$ & $-4,126069$ \\
\hline
\end{tabular}

A.4. Análisis de los residuos

\begin{tabular}{ccc}
\hline Normalidad & Correlación serial & Heterocedasticidad \\
\hline Jarque-Bera & LM & \\
\hline Joint & Lag 1 & Joint test \\
0,06 & 0,48 & 0,64 \\
\hline
\end{tabular}




\section{A.5 Raíz del polinomio característico}

Roots of Characteristic Polynomial

Endogenous variables: LNGP LNTAXMIX LNPIB LNRT

Exogenous variables: C DUM2002 DUM1989

Lag specification: 11

Date: 03/09/15 Time: 15:36

\begin{tabular}{cl}
\hline Root & Modulus \\
\hline 0,99653 & 0,99653 \\
0,92846 & 0,92846 \\
0,823744 & 0,823744 \\
0,576257 & 0,576257 \\
\hline
\end{tabular}

No root lies outside the unit circle.

VAR satisfies the stability condition.

\section{A.6. Análisis de descomposición de la varianza}

\begin{tabular}{cccccc}
\hline \multicolumn{5}{c}{ Variance Decomposition of LNGP: } \\
\hline Period & S.E. & LNGP & LNTAXMIX & LNPIB & LNRT \\
\hline 1 & 0.12 & 100.00 & 0.00 & 0.00 & 0.00 \\
\hline 5 & 0.24 & 43.24 & 0.03 & 56.53 & 0.20 \\
\hline 10 & 0.36 & 19.64 & 0.02 & 80.09 & 0.25 \\
\hline Period & S.E. & LNGP & LNTAXMIX & LNPIB & LNRT \\
\hline 1 & 0.13 & 10.74 & 89.26 & 0.00 & 0.00 \\
\hline 5 & 0.25 & 7.98 & 91.97 & 0.05 & 0.00 \\
\hline 10 & 0.30 & 6.97 & 92.83 & 0.21 & 0.00 \\
\hline & & Variance Decomposition of LNPIB: & \\
\hline Period & S.E. & LNGP & LNTAXMIX & LNPIB & LNRT \\
\hline 1 & 0.08 & 32.49 & 3.58 & 63.93 & 0.00 \\
\hline 5 & 0.22 & 7.92 & 2.06 & 89.94 & 0.08 \\
\hline 10 & 0.34 & 3.77 & 1.33 & 94.76 & 0.13 \\
\hline
\end{tabular}


Variance Decomposition of LNRT:

\begin{tabular}{cccccc}
\hline Period & S.E. & LNGP & LNTAXMIX & LNPIB & LNRT \\
\hline 1 & 0.16 & 35.67 & 0.22 & 25.05 & 39.05 \\
\hline 5 & 0.35 & 15.57 & 0.32 & 59.55 & 24.55 \\
\hline 10 & 0.48 & 9.23 & 0.38 & 74.98 & 15.41 \\
\hline
\end{tabular}

Cholesky Ordering: LNGP LNTAXMIX LNPIB LNRT

\section{REFERENCIAS BIBLIOGRÁFICAS}

Blanchard, O. \& Perotti, R. (2002). An Empirical Characterization of the Dynamic Effects of Changes in Government Spending and Taxes on Output, The Quarterly Journal of Economics, 117 (4), 1329-1368.

Enders, W. (1995). Applied Econometric Time Series, New York: John Wiley and Sons. Engle R. \& Granger C. W. (1987). Co-integration and Error Correction: Representation, Estimation and Testing, Econometrica, 55 (2), 251-276.

Martínez, C. (2015). Instituciones Políticas, Decisiones Fiscales y Resultados Económicos: el Caso de Argentina. Doctorado en Economía. Universidad Nacional del Sur.

Perotti, R. (2004). Estimating the Effects of Fiscal Policy in OECD Countries, IGIER: Università Bocconi, Working Paper, No. 276.

Rezk, E., Avramovich, M., \& Basso, M. (2006). Dynamic Effects of Fiscal Shocks upon diverse Macroeconomic Variables: A Structural VAR Analysis for Argentina. Trabajo presentado en XLI Reunión Anual de la Asociación Argentina de Economía Política. Salta, Argentina: AAEP. Recuperado de http://www.aaep. org.ar/anales/works/works2006/Rezk_Avramovich_Basso.pdf

Sims, C. (1980). Macroeconomics and Reality, Econometrica 48 (1), 1-48.

(C) 2016 por los autores; licencia otorgada a la Revista Estudios Económicos. Este artículo es de acceso abierto y distribuido bajo los términos y condiciones de una licencia Atribución-No Comercial 3.0 Unported (CC BY-NC 3.0) de Creative Commons. Para ver una copia de esta licencia, visite http://creativecommons.org/ licenses/by-nc/3.0/ 